\title{
A Facilitator Support System that Overlooks Keywords Expressing the True Intentions of All Discussion Participants
}

\author{
Chika Oshima ${ }^{1}$, Koichi Nakayama ${ }^{4}$ \\ Faculty of Science and Engineering \\ Saga University, Saga, Japan
}

\author{
Tatsuya Oyama ${ }^{2}$, Chihiro Sasaki $^{3}$ \\ Graduate School of Science and Engineering \\ Saga University, Saga, Japan
}

\begin{abstract}
This paper proposed the Keyword Movement Disclose System (KMDS), which allows a facilitator of discussion to watch a record of the moving keywords in a Discussion Board System (DBS). In the DBS, the discussion participants place each keyword in a box made for each item to be discussed. The keywords in the box were expected to show each participant's opinion and intention, because the participant's individual display was not disclosed to the other participants. Therefore, if the facilitator of the discussion can see the true opinions and intentions of all participants via the keywords in the boxes through the KMDS, the facilitator will be more appropriately advance the discussions and be able to draw conclusions based on diverse opinions. Moreover, the KMDS may contribute to the development of an artificial intelligence facilitator. In this paper, we conducted an experiment in which ten facilitators were asked to listen to a recorded discussion held by nine participants using the DBS. Five of the facilitators used the KMDS while listening the recorded discussion. It was suggested that KMDS may allow the facilitators to build a consensus from various viewpoints of the participants, although the results of the experiment did not show much difference depending on the conditions with/without KMDS.
\end{abstract}

Keywords-Keyword movement disclose system; discussion board system; facilitator; putting keywords in box

\section{INTRODUCTION}

Although diverse perspectives shared by employees enhance corporate competitiveness, the discussions that concentrate diverse values can easily become confused [1]. The team members who differ on information diversity must engage in high quality communication to reconcile differing approaches to task completion [2]. Team members with diverse information perform better when they exchange information effectively. In contrast, their performances become poor when they rely on their own limited perspective [3]. In such a case, a facilitator [4] can appropriately advance discussions based on various viewpoints [1].

There are several studies that have automated the role of facilitators by generating a facilitator's questioning based on pattern-matching rules [5], analyzing the words written on a bulletin board and automatically facilitating the discussion [6, 7]. Although there are many discussion support systems [8, 9], few automatically facilitate during discussions. Since the facilitator role is different from those of a secretary or moderator, it is not enough to recognize the utterances of the discussion participants by voice recognition and to collect the hot opinions.

The role of facilitators includes designing the process, controlling the process, organizing and inspiring discussions, and forming an agreement $[1,4]$. In this paper, we specifically focus on the fourth role. Sasaki [1] defines the fourth role as follows:

The facilitator elicits opinions from all participants as much as possible and gives a sense of conviction that the discussion was properly and adequately conducted. Identifying the right time, the facilitator encourages participants to reach conclusions and encourage consensus building.

We aim to develop an artificial intelligence (AI) facilitator that can autonomously facilitate discussions. The discussion addressed in this study calls for participants to form a collective consensus on a given set of problems and draw conclusions after considering various opinions [1, 10]. A competent facilitator reduces the peer pressure among participants and appropriately facilitates discussions based on different perspectives [1].

There are a lot of decision support systems that assist people by presenting information, knowledge, and analytical results: a system determines the best teacher using the $\mathrm{C} 4.5$ decision tree algorithm method [11], a system that suggests new fish that should be added to the aquarium tank based on the current environmental conditions of the aquarium [12], an automatic expert system that helps head of university department to choose lecturers and assign better course for them [13]. However, these systems do not help to make decision of the discussion.

"Wordy," which creates a word cloud based on lecture video content, allows a user to find the points they want to see in the video [14]. "Discussion Mining" generates structured data on discussion content semi-automatically and displays a graph structuralized with the pertinent information and keyword [15]. "Discussion Map" is a system, which supports consensus-building on multi-party conversations. Discussion participants themselves extract keywords during discussion and place them on the discussion map as nodes. The discussion is structured as a graph [16]. These systems allow the discussion participants to know the status of the discussion. 
The Discussion Board System (DBS), ver.1.0, ver. 2.0 [1], and ver. $2.1[10]$ has been developed to realize the role of a human facilitator. Before starting the discussion, items to be decided during the discussion are displayed at the top of each "box" in the DBS display. The DBS extracts nouns (called "keywords") from the participants' utterances and displays them. Each participant can put the keywords into a box according to their opinion and intention. Each participant's screen is invisible to other participants (a psychological safety zone [1] is one of the DBS's features); therefore, the true intentions of each participant will be expressed in the keywords they place in the boxes $[1,10]$.

The final decision made by the facilitator may change once they examine the keywords in each participant's box. How should the AI facilitator use the keywords in each participant's boxes to make a final decision? In this paper, ten facilitators listened to a discussion using the DBS, with/without watching a record of the keywords that showed when and who put each keyword into each box. Then, the final decisions the facilitators made while watching the record are compared with those made without watching the decisions.

\section{EXPERIMENTAL METHOD}

\section{A. Overview}

Fig. 1 shows an overview of the experiment. Ten male university students (Facilitators A-J) participated. They were paid a small reward to compensate them for their time. They were asked to listen to a recorded discussion in which nine other students (Participants O-W) discussed a fictional scenario. The participants belong to the same laboratory as Facilitators A-J. During the discussion, each participant used DBS ver. 2.1 (see Section IIB) on his own personal computer. Half of the facilitators, while listening to the discussion, were also watching the Keyword Movement Disclose System (KMDS) (see Section IIC), alerting them to when and who put each keyword in the box. After listening to the discussion, the facilitators answered questions.

\section{B. Discussion Board System ver. 2.1}

Fig. 2 indicates the display on DBS ver. 2.1 [2] that The participants used during the discussion. The boxes for each item to be discussed are displayed in a category area. Each participant puts the keywords extracted from their utterances into the box, based on their opinion and intention. The participants cannot see each other's category areas. If all participants in the discussion put the same word in the same box, the word's color changes to green. A participant can put any word in the parking area while debating whether or not to put it in the box. The comment area presents users with comments, in particular, ones that encourage those who have not spoken or moved any words on the screen for a certain period of time to join in the discussion.

\section{Keyword Movement Disclose System}

Half of the facilitators in this paper used KMDS to watch as keywords were moved, and they had access to data showing when and who, among the nine participants, put each keyword into a box. Fig. 3 shows the KMDS display. The top left of the screen shows the remaining time until the discussion will end, because this research assumes that company meetings feature discussion times that are set in advance. The top right area of the screen shows a timeline of the keywords' movement, and indicates when and who moved what keyword to which box. For example, one of the participants added the keyword " 3 hours" to the item "performance time" when 48 minutes and 27 seconds remained for the discussion. The lower half of the window, in the category area, shows what kind of keyword each word is and how many participants moved it to each box. Although the DBS does not allow the discussion participants to see each other's category areas, the KMDS discloses the movements of the keywords of all participants to the facilitator.

KMDS usually displays these keyword movements in real time; however, in the experiment detailed in this paper, a prerecorded discussion and a pre-prepared record of keyword movements in KMDS were used.

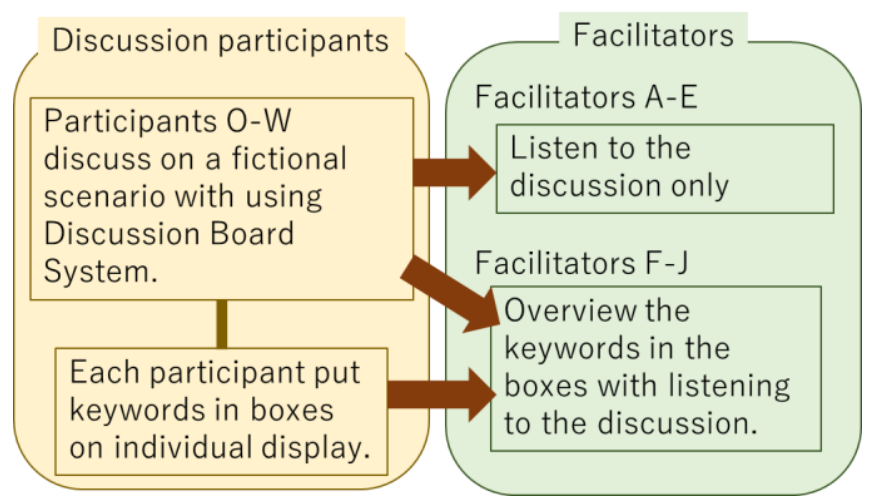

Fig. 1. Overview of the Experiment.

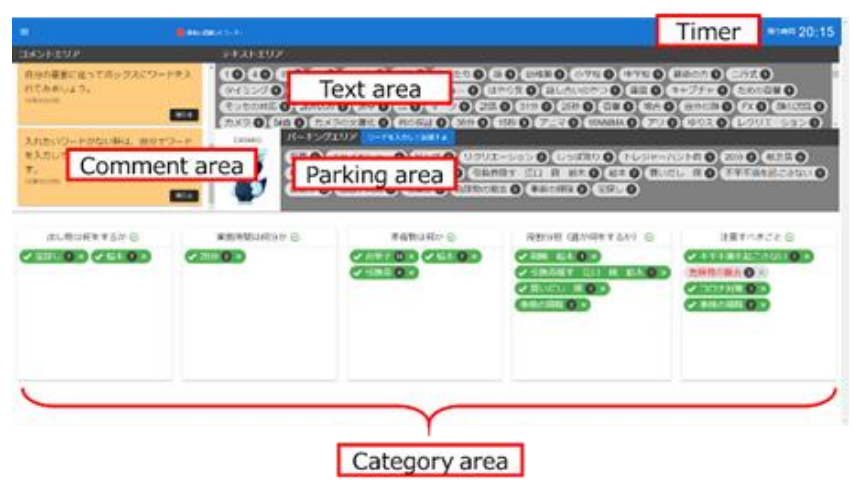

Fig. 2. Discussion Board System ver. 2.1 [1].

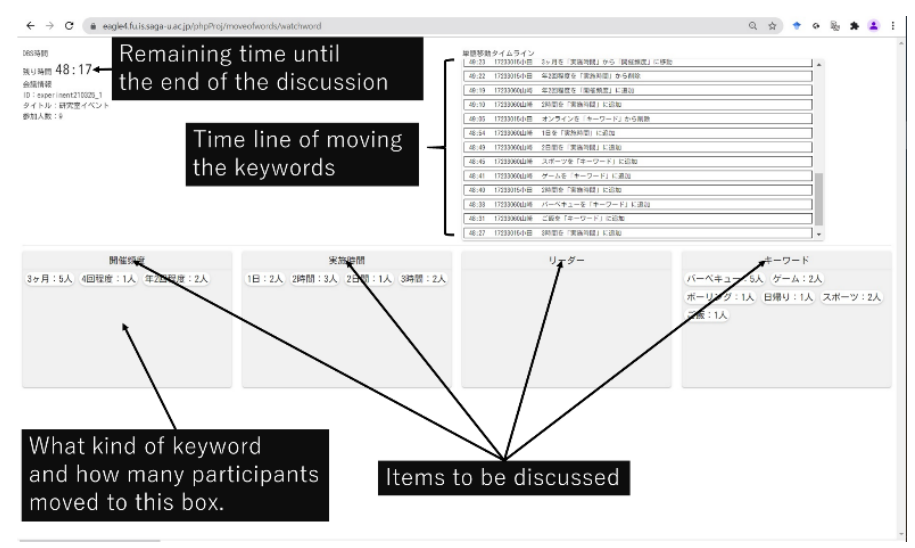

Fig. 3. Keyword Movement Disclose System. 


\section{Method}

Each facilitator was asked to listen to a prepared one-hour discussion. The instructions for the facilitators were as follows:

From now on, you will listen to the recording of a discussion by nine people. They are discussing a "social event" to be held this year to build the relationships among the members of the laboratory. In the one-hour discussion, there are four items to decide.

Item 1: Frequency of holding the event

Item 2: Length of time per event

Item 3: Elect one leader and one sub-leader to put on the social event.

Item 4: Select two or three keywords that represent the social event from the following:

training camp / travel / welcome party / exchange party / game / sports / movie / birthday party / tournament / meal / drinking party / BBQ / festival / camping / online / other (write down here).

You are allowed to take notes on your smartphone and/or paper. The family names of the nine members are as follows:

Participants O, P, Q, R, S, T, U, V, and W (real names were indicated).

You are assumed to be a senior employee, compared to these nine discussion participants, and you have the authority to make final decisions on these four items. Listen to the discussion and come to the conclusion that you think is best for the laboratory. You are not someone who simply concludes an agreement or a secretary taking notes. Think about what the best conclusion is based on the discussion.

The KMDS was always displayed on half of the facilitators' computer screens, along with the following instructions for Facilitators F-J:

A record of the nine participants' moved words in a discussion support system appears on the KMDS screen in chronological order. Press the start button at the same time as the start button for the recording. Watch this screen while listening to the audio recording.

The facilitators were informed in advance that they would be asked what conclusion they consider to be the best for the laboratory concerning each of the four items after listening to the discussion.

After listening to the discussion, we will use a questionnaire to ask what conclusion you consider to be the best for the laboratory for each item, the reasons for your conclusions, and your degree of certainty that the conclusions are good ones.

In the recording, Participants $\mathrm{O}-\mathrm{W}$ had discussed "a social event to be held this year to build the relationships among the members of the laboratory." The participants were asked to decide the same items as above four items during the discussion.

\section{E. Questionaire}

The questionnaire asked the following:

Question 1-1: Please draw the conclusion that you think is the best for the laboratory about "Item 1: Frequency of holding the event."

Question 1-2: Please explain why you drew this conclusion.

Question 1-3: Please indicate how confident you are of the suitability of the conclusion you made (5: very confident -1 : not sure at all).

Question 2-1: Please draw the conclusion that you think is the best for the laboratory about "Item 2: Length of time per event."

Question 2-2: Please explain why you drew this conclusion.

Question 2-3: Please indicate how confident you are of the suitability of the conclusion you made (5: very confident -1 : not sure at all).

Question 3-1: Please draw the conclusion that you think is the best for the laboratory about "Item 3: Elect one leader and one sub-leader to put on the social event."

Question 3-2: Please explain why you drew this conclusion.

Question 3-3: Please indicate how confident you are of the suitability of the conclusion you made (5: very confident -1 : not sure at all).

Question 4-1: Please draw the conclusion that you think is the best for the laboratory about "Item 4: Select two or three keywords that represent the social event from the following."

Question 4-2: Please explain why you drew this conclusion.

Question 4-3: Please indicate how confident you are of the suitability of the conclusion you made (5: very confident -1 : not sure at all).

Question 5: Please tell me as much as you can about what kind of situation, intention/opinion you think Participant $U$ had in the discussion.

\section{RESULTS}

\section{A. Conclusions by the Participants and Facilitators}

Fig. 4 to 7 show the rates of conclusions that the participants considered and which ones remained in each box; they also indicate whether the facilitators were working with/without KMDS on each item. Each X-axis of the figures indicates the answers. Each y-axis of the figures shows the ratio of the number of responses to the number of the participants/facilitators.

Fig. 4 shows the results of the conclusion on Item 1 (Question 1-1), "Frequency of holding the event." Although one of the facilitators without KMDS considered "once every one or two weeks" to be the best conclusion for the laboratory, the other answers and remaining keywords in the box for Item 1 were "once every three months." 


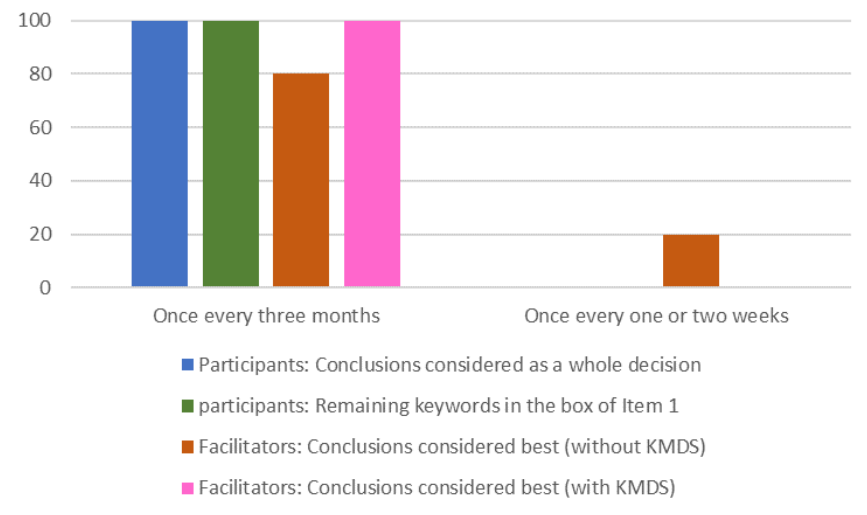

Fig. 4. Results of the Conclusion on Item 1.

Fig. 5 shows the results of the conclusion on Item 2 (Question 2-1), "Length of time per event." Although "two or three hours" and "three hours" were the most considered conclusion by the participants, "One day" was the most-often remaining among the keywords in the box for Item 2 and considered to be the best conclusion by the facilitators without KMDS.

Fig. 6 shows the results of the conclusion on Item 3 (Question 3-1), "Elect one leader and one sub-leader to put on the social event." All participants considered "Participant T is the best for the leader of this event" to be the conclusion of this discussion. In contrast, opinions on appropriate sub-leaders were divided among participants $\mathrm{P}, \mathrm{Q}$, and $\mathrm{R}$. About half of the participants selected Participants Q or R, while most facilitators without KMDS concluded in favor of Participant R. Furthermore, the facilitators with KMDS, instead of giving the participants' names, based their conclusions on personal opinions, such as "participants who live near the university" (Facilitator F), "the sub-leader is expected to be a different year from the leader" (Facilitator F), and "everyone should take turns" (Facilitator $\mathrm{H})$.

Fig. 7 shows the results of the conclusion on Item 4 (Question 4-1), "Select two or three keywords that represent the social event from the following." Most participants and facilitators concluded "game (online)." However, the keywords left in each participant's box varied.

\section{B. Reasons for the Conclusions}

This section focus on reasons for the conclusions. We classified the reasons (answers for Questions 1-2, 2-2, 3-2, and 4-2) into six types:

1) Drew the same conclusions and reasons as the majority of the participants.

2) Although they drew the same conclusions as the majority of discussion participants, the reasons given for the conclusions were different from the reasons the participants gave.

3) Drew the same conclusions and reasons as a minority of discussion participants.

4) Although they drew the same conclusions as a minority of discussion participants, the reasons for these conclusions were different from those given by the participants.
5) Although the conclusion was not based on the participants' comments during the discussion, the reason for the conclusion was same as what someone said.

6) The conclusions and reasons did not come from what anyone had said.

Table I shows the results after classifying the reasons for the conclusions, according to the above six types, and the results of the facilitators' confidence in their conclusions. There were no significant differences between the results of the conditions, without and with KMDS. However, in the reasons for Question 3 (answering Question 3-2), Participants F and H described why each of them did not write down the specific names of the participants (see Fig. 7) as follows:

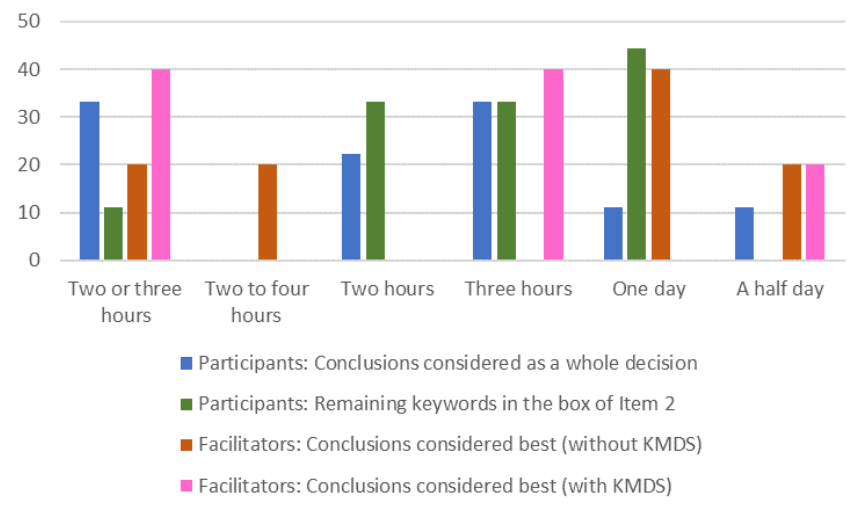

Fig. 5. Results of the Conclusion on Item 2.

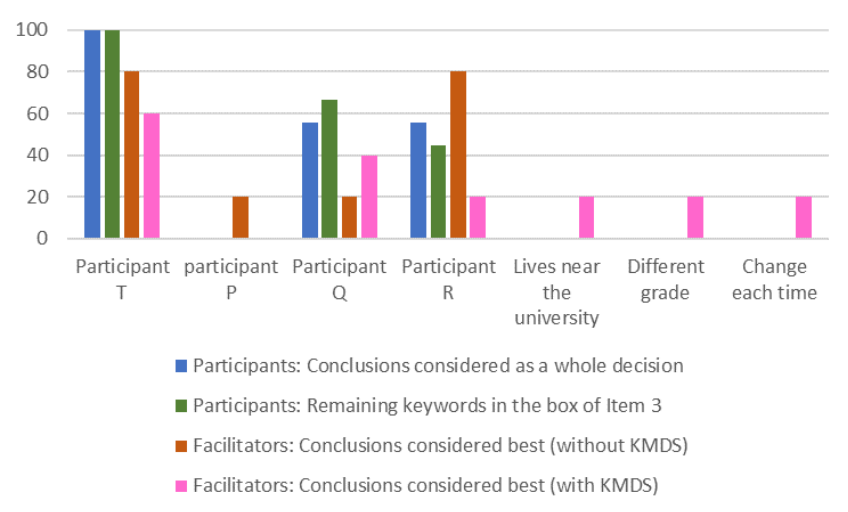

Fig. 6. Results of the Conclusion on Item 3.

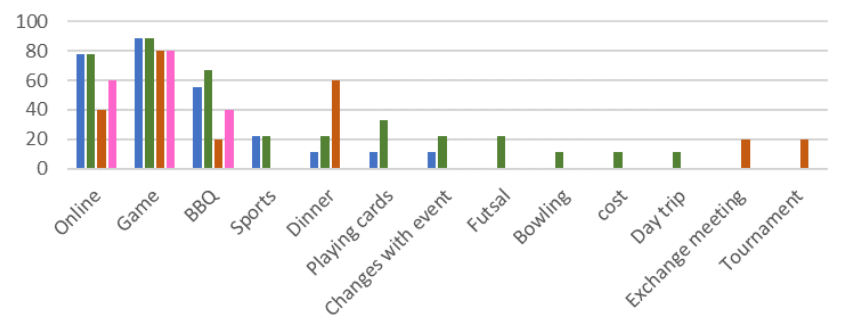

- Participants: Conclusions considered as a whole decision

- participants: Remaining keywords in the box of Item 4

- Facilitators: Conclusions considered best (without KMDS)

nacilitators: Conclusions considered best (with KMDS)

Fig. 7. Results of the Conclusion on Item 4. 
Facilitator F: I did not write the specific name because I felt the discussion was a little forced. But it seems that this (Participant F's conclusion) was the direction of the participants' discussion. Moreover, I considered that it is this method (a participant who lives near the university, the years of leader and the sub-leader are different) that the decision on the event's content and communication among the participants proceeded smoothly (when they will hold the laboratory's event).

Facilitator $\mathrm{H}$ : The conclusions of the participants were basically a majority vote, but I felt that the consent of the participants was not so much obtained, and that some imposed their views on others. Some participants were concerned about the time for research presentations, classes, and job hunting. So, I think it is best for the participants with plenty of time to take charge in turn, according to the situation of each participant, rather than having one person takes charge.

\section{Guessing Participant U's Opinions and Intentions}

The results of Question 5, where the facilitators without KMDS were asked about Participant $\mathrm{U}$ were as follows:

Facilitator A: She (Participant $U$ ) wanted to match the faced and named of the laboratory members.

Facilitator B: Since she was just assigned to the laboratory, she thought they should have an opportunity to interact as much as possible.

Facilitator C: She wants to match the names with the faces of everyone in the laboratory.

Facilitator D: She was trying to make the meeting go smoothly by giving affirmative opinions.

Facilitator E: She was difficult to get into the discussion, because the story of the discussion changed a lot in the second half of the discussion.

The results of Question 5 where the facilitators had access to KMDS were as follows:

Facilitator F: She would like to try to match the names and faces of the laboratory members.

Facilitator G: I do not know who Participant $U$ was.

Facilitator H: Basically, she made statements as prompted, and she did not have a positive opinion. Although she had enough opportunities to speak, she was a bystander. She was not interested in the conclusions of this discussion.

Facilitator I: She was not very enthusiastic about the event itself.

Facilitator J: Since she said that she could not match the laboratory members' names and faces, I think it was difficult for her to speak in this discussion where there were many senior members.

In fact, Participant $U$ said that she wanted to match the names and faces of the laboratory's staff members. She spoke very little. She left the keywords in the box for Item 4, BBQ, sports, game, and online.
TABLE I. RESULTS OF REASONS AND CONFIDENCE

\begin{tabular}{|c|c|c|c|c|c|}
\hline & & \multicolumn{2}{|c|}{ Question 1} & \multicolumn{2}{|c|}{ Question 2} \\
\hline & & $\begin{array}{l}\text { Reason } \\
\text { (six types) }\end{array}$ & $\begin{array}{l}\text { Confidence } \\
(1-5)\end{array}$ & $\begin{array}{l}\text { Reason } \\
\text { (six types) }\end{array}$ & $\begin{array}{l}\text { Confidence } \\
(1-5)\end{array}$ \\
\hline \multirow{6}{*}{$\begin{array}{l}\text { Without } \\
\text { KMDS }\end{array}$} & $\boldsymbol{A}$ & 1 & 4 & 4 & 3 \\
\hline & $B$ & 5 & 5 & 2 & 4 \\
\hline & $C$ & 2 & 4 & 4 & 3 \\
\hline & $D$ & 2 & 4 & 4 & 4 \\
\hline & $E$ & 1 & 5 & 2 & 4 \\
\hline & Avg. & - & 4.4 & - & 3.6 \\
\hline \multirow{8}{*}{$\begin{array}{l}\text { With } \\
\text { KMDS }\end{array}$} & $F$ & 1 & 4 & 4 & 4 \\
\hline & $G$ & 1 & 5 & 2 & 5 \\
\hline & $H$ & 2 & 4 & 2 & 4 \\
\hline & $I$ & 1 & 4 & 1 & 4 \\
\hline & $J$ & 1 & 5 & 2 & 4 \\
\hline & Avg. & - & 4.4 & - & 4.2 \\
\hline & & \multicolumn{2}{|c|}{ Question 3} & \multicolumn{2}{|c|}{ Question 4} \\
\hline & & $\begin{array}{l}\text { Reason } \\
\text { (six types) }\end{array}$ & $\begin{array}{l}\text { Confidence } \\
(1-5)\end{array}$ & $\begin{array}{l}\text { Reason } \\
\text { (six types) }\end{array}$ & $\begin{array}{l}\text { Confidence } \\
(1-5)\end{array}$ \\
\hline \multirow{6}{*}{$\begin{array}{l}\text { Without } \\
\text { KMDS }\end{array}$} & $\boldsymbol{A}$ & 2 & 4 & 2 & 5 \\
\hline & $B$ & 2 & 4 & 2 & 5 \\
\hline & $C$ & 2 & 4 & 1 & 4 \\
\hline & $D$ & 2 & 4 & 2 & 4 \\
\hline & $E$ & 4 & 4 & 2 & 4 \\
\hline & Avg. & - & 4.0 & - & 4.4 \\
\hline \multirow{6}{*}{$\begin{array}{l}\text { With } \\
\text { KMDS }\end{array}$} & $F$ & 6 & 5 & 2 & 3 \\
\hline & $G$ & 2 & 5 & 2 & 4 \\
\hline & $\boldsymbol{H}$ & 6 & 3 & 6 & 4 \\
\hline & $I$ & 2 & 3 & 1 & 4 \\
\hline & $J$ & 2 & 4 & 2 & 3 \\
\hline & Ave. & - & 4.0 & - & 3.6 \\
\hline
\end{tabular}

\section{DISCUSSION}

More innovation will occur if the minority has a high level dissent and is highly involved in team decision making [17]. However, words spoken by multiple participants and/or at the end of a discussion often become a final conclusion if it is not possible to examine various opinions among the participants. A facilitator is responsible for avoiding such situations. If an AI facilitator only statistically analyzes and acquires the words that are spoken many times and adopts the opinions of the majority as a conclusion, it can result in abandoning various opinions.

Participants in the experiment's discussions believed that they had reached a conclusion on the items to be decided; however, and especially related to the items concerning the election of a sub-leader and the events themselves, no single conclusion was drawn. Most facilitators in the experiment listened to the discussion and drew their conclusions based on the opinion of the majority. However, two of the facilitators 
who listened to the discussion while also using KMDS did not name a specific participant as sub-leader. One of the facilitators presented the ideal characteristics of leaders and sub-leaders, such as "people who live near the university" and "leaders and sub-leaders who are in different years." An examination of the keywords in the box makes it clear that the participants' opinions were not unified; moreover, Facilitator F must have noticed that the leader and sub-leader candidates were in different years. That fact may have led to the idea that leaders from different years are better for staging a successful event. Another facilitator concluded that "everyone takes turns at each event." He did not think that the participants' consent was much obtained about the leader and the sub-leader, unlike other facilitators.

The keywords that Participant $\mathrm{U}$ left in the box were not significantly different from that of other participants. However, she simply put each keyword in the box in the direction of the discussion of the entire participant, and did not seem to actively express her own opinions in the keywords. By comparing the timing at which a participant puts each keyword in a box with other participants, it may be possible to estimate the participant's degree of participation and agreement in the discussion.

In this way, the facilitators using KMDS could confirm the true opinions of all the participants based on the keywords they placed in the boxes. The facilitators could also know that the participants did not reach an agreement. Hence, it was suggested that the facilitators may be able to draw conclusions in a different direction from those available to the participants.

Although the results of the experiment did not show much difference based on the conditions (with/without KMDS), if the participants have more diverse ideas and a firmer hierarchical relationship, the usefulness of KMDS may have been further demonstrated. It is also undeniable that there were some differences in the qualities of individual facilitators.

Currently, the AI facilitator of our research may simply draw conclusions based on the majority keywords. In the future, the AI facilitators should also consider the lack of consensus, the presence of minority keywords, and the keywords that were mentioned only at the beginning of the discussion [18]. In some cases, the AI facilitator may need to present some new keywords that encourage a change in thinking. For example, when the sub-leader was not easily decided, the idea of "Everyone takes turns being in charge" was given by Facilitator H. In this way, skillful facilitators can also lead the participants to a desired conclusion $[19,20]$.

\section{CONCLUSION}

This paper proposed a Keyword Movement Disclose System (KMDS) that displays when and who puts a keyword into which box in a discussion support system (DBS). Ten facilitators listened to a discussion with/without KMDS. In some results, there were subjective differences in the conclusions drawn by the discussion participants and the facilitators, and between the facilitators with/without access to KMDS. The facilitators using KMDS could see the true opinions of all participants being expressed as they moved keywords into the boxes.
In the future, we will develop an AI facilitator which is able to appropriately advance the discussions based on various viewpoints and encourage consensus building, not just show the results of a majority vote.

\section{REFERENCES}

[1] C. Sasaki, T. Oyama, S. Kajihara, C. Oshima, and K. Nakayama, "Online Discussion Support System with Facilitation Functio," International Journal of Advanced Computer Science and Applications, vol. 12, no. 8, paper 37, 2021.

[2] S. L. Marlow, C. N. Lacerenza, and E. Salas, "Communication in virtual teams: A conceptual framework and research agenda," Human Resource Management Review, vol. 27, no. 4, pp. 575-589, 2017

[3] A.C. Homan, D. Van Knippenberg, G.A. Van Kleef, and C.K. De Dreu, "Bridging faultlines by valuing diversity: diversity beliefs, information elaboration, and performance in diverse work groups," Journal of Applied Psychology, vol. 92, no. 5, pp. 1189, 2007.

[4] T. Mori, Facilitator training course. Japan: Diamond, Inc., 2007. (in Japanese).

[5] Y. Ikeda, and S. Shiramatsu, "Generating questions asked by facilitator agents using preceding context in web-based discussion," In 2017 IEEE International Conference on Agents, pp. 127-132, July 2017.

[6] T. Ito, S. Suzuki, N. Yamaguchi, T. Nishida, K. Hiraishi, and K. Yoshino, "D-Agree: Crowd Discussion Support System Based on Automated Facilitation Agent," Proc. of the AAAI Conference on Artificial Intelligence, vol. 34, no. 09, pp. 13614-13615, 2020.

[7] J. Haqbeen, T. Ito, S. Sahab, R. Hadfi, S. Okuhara, N. Saba, et al., "A contribution to covid-19 prevention through crowd collaboration using conversational AI \& social platforms," arXiv preprint arXiv:2106.11023, 2021.

[8] R. Schroeter, J. Hunter, and D. Kosovic, "Vannotea: A collaborative video indexing, annotation and discussion system for broadband networks," Proceedings of the Second International Conference on Knowledge Capture: K-Cap, 2003.

[9] H. Tomobe and K. Nagao, "Discussion ontology: knowledge discovery from human activities in meetings," In Annual Conference of the JSAI, pp. 33-41, Springer, Berlin, Heidelberg, June 2006.

[10] T. Oyama, C. Sasaki, C. Oshima, and K. Nakayama: AI Facilitator Allows Participants to Conduct a Friendly Discussion and Contribute to Feasible Proposals, Communications in Computer and Information Science, vol. 1420, pp.523-530, 2021.

[11] H. Siahaan, H. Mawengkang, S. Efendi, A. Wanto, and A. P. Windarto, "Application of classification method C4. 5 on selection of exemplary teachers," Journal of Physics: Conference Series, IOP Publishing, vol. 1235, no. 1, pp. 012005, 2019.

[12] M. Mohammadi, and S. Jafari, "An expert system for recommending suitable ornamental fish addition to an aquarium based on aquarium condition," arXiv preprint arXiv:1405.1524, 2014.

[13] S. Fekri-Ershad, H. Tajalizadeh, and S. Jafari, "Design and Development of an Expert System to Help Head of University Departments," International Journal of Science and Modern Engineering, vol. 1, no. 2, pp. 45-48, 2013.

[14] W. Zhu, J. Zang, and H. Tobita, "Wordy: Interactive Word Cloud to Summarize and Browse Online Videos to Enhance eLearning," 2020 IEEE/SICE International Symposium on System Integration, IEEE, pp. 879-884, 2020.

[15] K. Nagao, K. Kaji, D. Yamamoto, and H. Tomobe, "Discussion mining: Annotation-based knowledge discovery from real world activities," Pacific-Rim Conference on Multimedia, Springer, Berlin, Heidelberg, pp. 522-531, 2004.

[16] R. Kirikihira, and K. Shimada, "Discussion map with an assistant function for decision-making: A tool for supporting consensusbuilding," International Conference on Collaboration Technologies, Springer, Cham. pp.3-18, 2018.

[17] C. K. De Dreu, and M. A. West, "Minority dissent and team innovation: The importance of participation in decision making," Journal of applied Psychology, vol. 86, no. 6, pp. 1191, 2001. 
[18] K. Nishimoto, Y. Sumi, R. Kadobayashi, K. Mase, and R. Nakatsu, "Group thinking support with multiple agents," Systems and Computers in Japan, vol. 29, no. 14, pp. 21-31,1998.

[19] T. Proctor, "Creative problem-solving techniques, paradigm shift and team performance," Team Performance Management: An International Journal, vol. 26, no. 7/8, pp. 451-466, 2020.
[20] S. Ikari, Y. Yoshikawa, and H. Ishiguro, "Multiple-Robot Mediated Discussion System to support group discussion," 29th IEEE International Conference on Robot and Human Interactive Communication, IEEE, pp. 495-502, 2020. 